\title{
Analysis and Design of Clinical Information Management System Based on UML of AIDS of Traditional Chinese Medicine
}

\author{
Songhe Shi ${ }^{[1,2]}$ \\ ${ }^{1}$ School of Computer Science \& Technology \\ Wuhan University of Technology \\ Wuhan, China \\ ${ }^{2}$ College of Public Health, Zhengzhou University \\ Zhengzhou, China \\ zzussh@126.com
}

\author{
Shengwu Xiong ${ }^{1}$ \\ ${ }^{1}$ School of Computer Science \& Technology \\ Wuhan University of Technology \\ Wuhan, China \\ xiongsw@whut.edu.cn
}

\begin{abstract}
Purpose] In order to find out AIDS syndrome discipline of traditional Chinese medicine, and so as to conduct dialectical analysis scientifically to patients, and also lay the foundation for the Chinese medicine diagnosis and treatment of AIDS, and combined with the situation of Henna AIDS Chinese medicine prevention and cure at the same time, we develop AIDS traditional Chinese medicine clinical information management system.[Methods]Employ unified modeling language (UML) as the modeling tool of the system. Based on Rational unified process (RUP) development, ID number as patients' recognition number. [Procedure] According to the characteristics of AIDS TCM clinical syndrome, this article has deeply introduced the application of UML in the analysis and design of software, it also used iterative type development process of use case drive, and explained the system analysis and design process in detail at the same time. Combined with an instance of the UML diagrams, we introduced the application of UML software modeling in detail. [Results]: Combined with nation TCM treatment AIDS projects and Henan Province AIDS TCM prevention experience, the management system frame and model of the clinical syndrome of AIDS in Chinese traditional medicine have been constructed. According to the above model, we built the information management system of TCM clinical syndrome of AIDS comprehensive prevention and control. [Conclusion]UML modeling can speed up the development speed and improve system reliability. We have put traditional Chinese medicine syndromes basic information data of 1654 AIDS patients into the system, and have made the corresponding statistical analysis in view of the investigation data. We have provided practical and efficient work platform among doctors, patients and scientific researchers. The systematization and standardization of patients management have been realized, the dynamic management of information of patients' each stage has been realized, which will bring a beneficial effect on the systematical treatment of AIDS.
\end{abstract}

Keywords-AIDS; Traditional Chinese Medicine Syndrome; Traditional Chinese Medical science clinic information; UML

\section{INTRODUCTION}

There are no effective drugs and therapies to treat AIDS so far in the international medical community. Of many methods in the prevention and treatment of HIV/AIDS, the effect of treating AIDS in clinical experiment with traditional Chinese medicine is remarkable ${ }^{[1-3]}$. Therefore, in order to collect, manage and use information expediently, we combined with AIDS projects of nation TCM treatment and the experience of Henan Province AIDS traditional Chinese medicine prevention, the management system frame and model of the clinical syndrome of AIDS in Chinese traditional medicine have been constructed. According to the above model, we built TCM clinical information database, and collected aspects of data information such as patients' basic information, clinical manifestations, traditional Chinese medicine diagnosis and treatment method and so on. To develop investigation and study of AIDS clinical syndromes, bring into quantitative index; use biological statistical methods to analyze, in order to systematically study the occurrence, development and evolution law of AIDS, and this provide the foundation for establishing and confirming AIDS traditional Chinese medicine syndromes distribution rule and syndromes standards; and at the same time the foundation for dialectical theory of traditional Chinese medicine in treating AIDS can be laid; and provide a scientific basis for the policy-making of the government prevention of STD(AIDS), and also provide the foundation for the structure of the medical system and traditional Chinese medicine treatment of AIDS.

\section{The Design of Traditional Chinese Medical SYNDROME CLINICAL INFORMATION MANAGEMENT SYSTEM}

\section{A. Analysis Model}

To set up analysis model according to the demand analysis, at the same time, establishing the analysis model is also a further analysis of demand, analysis model mainly includes use case diagram and interaction diagram ${ }^{[4]}$, the use case diagram is a static modeling mechanism of RUP, while the interaction diagram is a dynamic modeling mechanism of RUP ${ }^{[5]}$.

\section{1) Use case diagram}

Use case is the method of capturing demand, use case diagram defines the framework and boundary of system, makes models for the behavior of future system, preliminary determines the system structure of the future. Use case diagram 
illustrates information completely in vernacular way, to help developers have a clearer understanding of the system behavior. In this paper, only take the use case diagram of user management modules for instance, as shown in Figure 1.

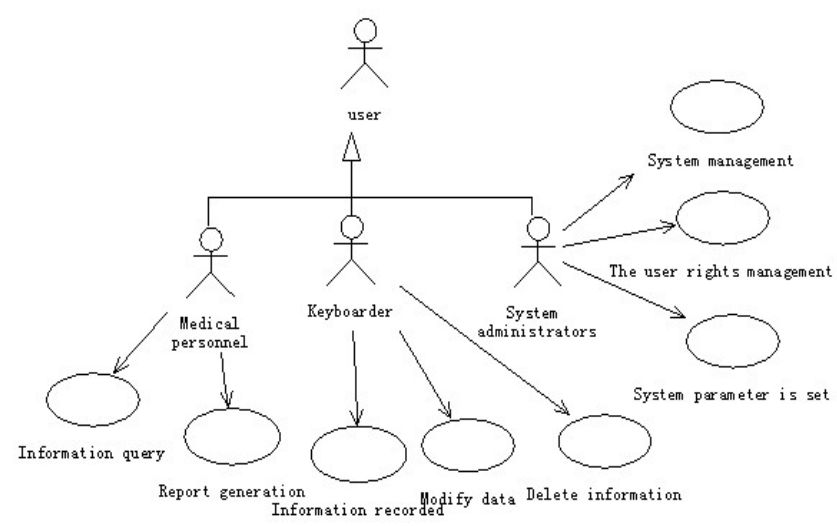

Figure 1. System use case diagram

\section{2) Interaction diagram}

Interactive diagram describes how the cases realize the interaction among objects; it is used for establishing the system dynamic behavior model. After analyzing and mapping use case diagram, it is necessary to analyze the main use case interactive behavior. We can understand the behavior of the cases much more clearly, further adjust the case view, and determine solutions of problems.

There are two kinds of interactive diagrams: sequence diagram and collaboration diagram. Sequence diagram is used to demonstrate the relationships between objects, and also emphasizes the chronological order of the messages between objects, and at the same time shows the interaction between objects; Collaboration diagram is used to describe the consummation of the identified transactions, and to describe the structural relationship of the massages connection between each objects. Sequence diagram emphasizes the chronological order of the messages, but it does not explicitly express the relationships between objects; Collaboration diagram emphasizes the organizational relationships of the objects involved in the interaction, the chronological order can be obtained from the sequence number. Sequence diagram and Collaboration diagram are semantically equivalent, they can be converted each other.

This system chooses sequence diagram. Sequence diagram analyze process flow of use case and the activities according to the order, take functions of inquiring the patient information for example, the sequence diagram can be explained vividly, as shown in Figure 2.

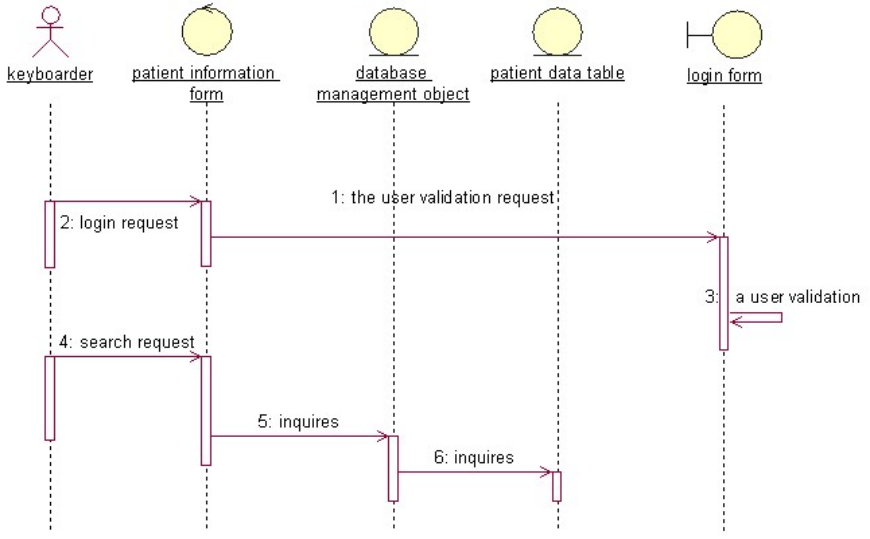

Figure 2. The sequence diagram of inquire the patient information

Sequence diagram shows the time sequence of message passing and function realization between objects. The figure expresses the meaning followed:

In this sequence diagram, there are five objects: keyboarders, login screen, patient information window, database management object, patient date tables.

"Inquires the patient information" process is as follows: keyboarders login firstly, and enter the patients' information window after checking, then input the query constraint. The query is sent to database management object, and the object inquires the information of patients, at last the query result will be returned to patients' information window. In figure 3 we can see that with time (lifeline) extension, the news abides by time order, which reflects the interactive processes between keyboarders and various other objects. Interaction diagram drawn here is mainly to verify the result of demand analysis and the correctness of use cases view, in the dynamics modeling process of the following model designed, the interactive diagram should be made a further analysis, clarification and improvement.

\section{B. Design Model}

After demand analysis, we can get the system concept model. In the design phase, we can capture design model from concept model. It includes class diagrams, object graphs, state charts, and activity diagrams. These class diagrams and object charts describe static structure of system; which are the static modeling mechanism of RUP. Activity diagrams and state charts describe the dynamic structure of system; which are the dynamic modeling mechanism of RUP.

\section{1) Class diagram}

A use case realization is probably completed by many analysis classes collaboratively, and an analysis class might be involved in various use cases realization. Class diagram indicates the static structure of system, describes static relationship between classes. It not only shows the structure of system, but also describes the behavior of system. Management system function modules are a little more, here take questionnaires management function module as an example for analysis. The main areas of this module are as follows: "entry questionnaire" class, "inquires the patient information" class, "changed the patient information" class, "delete patient 
information” class, etc. The main classes of this module are: boundary class (entry questionnaire interface class, inquires questionnaire interface class, modify questionnaire survey interface class, and delete questionnaire interface class), control class (entry of questionnaire class, inquires of questionnaire survey class, modification of questionnaire class, delete questionnaire categories) and entity class (questionnaire class). These classes and their relations are shown in Figure 3.

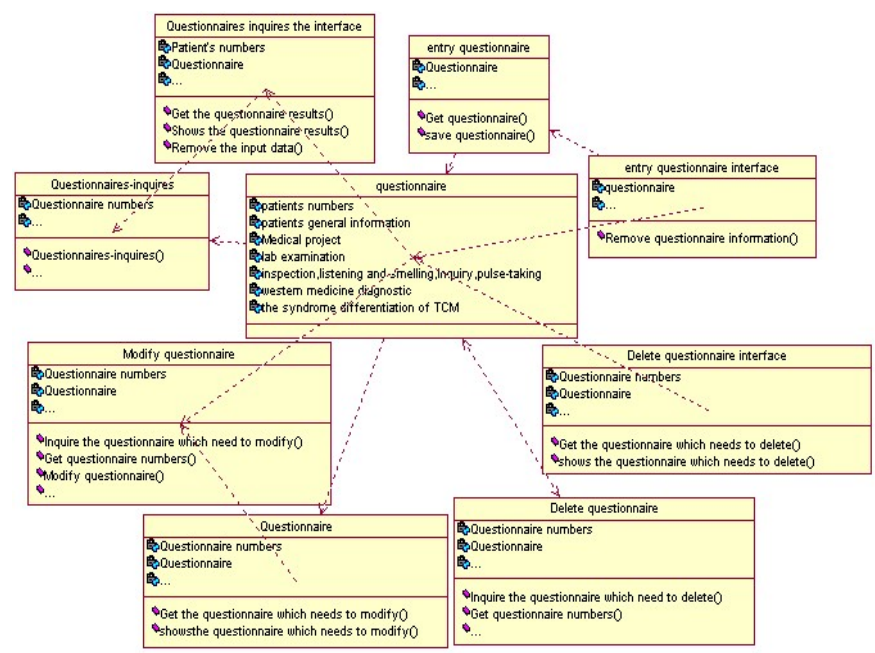

Figure 3. Management module UML class diagram

\section{2) Component diagram}

Component is a physical, replaceable part, which confirms to a group of interface in the system and provides compliance to its realization. A component diagram shows a group of components as well as their relationship, including compilation, link or the dependencies between components in the execution. Here take the component diagram of entering the personnel end for example to illustrate the UML component figure, as shown in Figure 4.

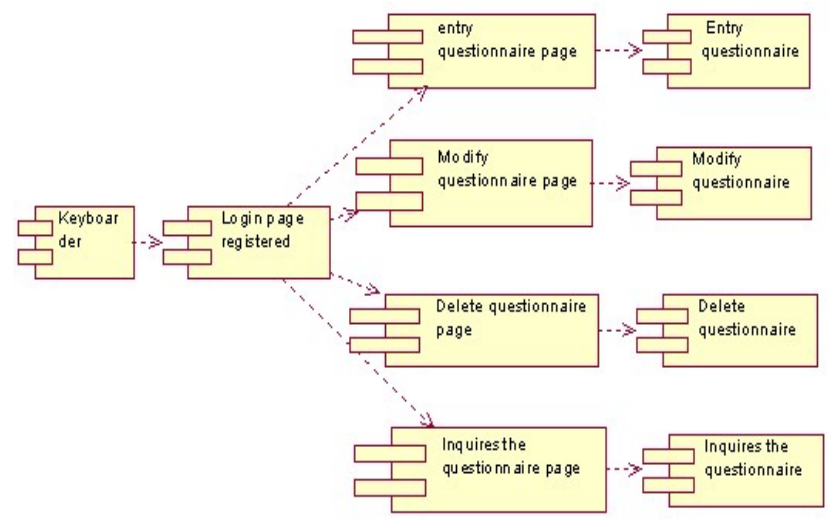

Figure 4. Construct figure of entry personnel end

UML component diagram shows the dependence of the various components in the system, the meaning expressed is as following:

Firstly, the keyboarder end enter the "login registered interface”, and reach to kinds of function interfaces; then into each corresponding management process; according to the function different, the interface and treatment are different. The figure also provides help for the other stages of the task, the details are as follows:

Assemble test case design during testing plan of demand analysis stage;

Specific design module interface during the design phase;

Design executable files and other components during coding phase;

Systematically check the verification standard during testing phase.

\section{3) Activity diagram}

Activity diagrams indicate the task execution in some process, and can also indicate the statement execution in algorithm process. In cases of analysis, it can use activity diagram to build model according to the process of use case, describes the specific working process. These activities are corresponding to each step in a use case. Modify questionnaire activity diagrams. As shown in Figure 5.

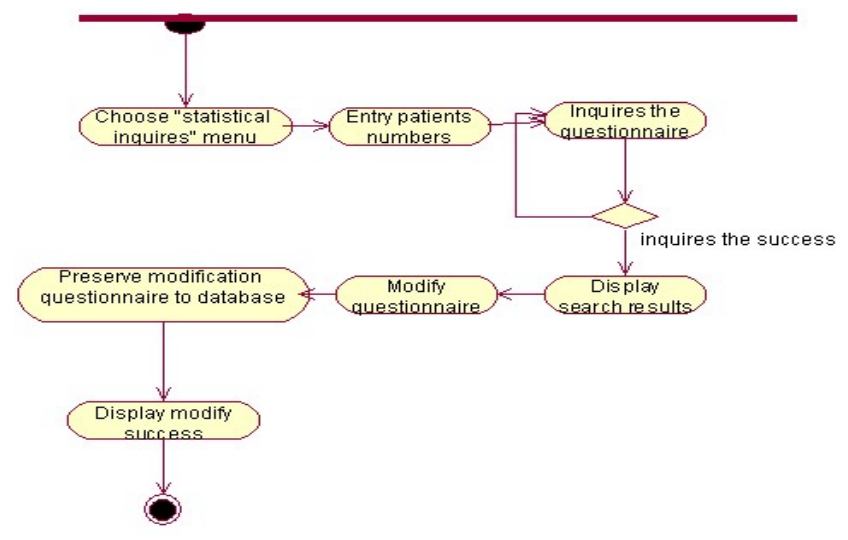

Figure 5. Modify questionnaire activities figure

In UML activity diagram above, the working process of the system realization of modifying questionnaire activities is performed. Participants of the activity were keyboarders; it described each action of the whole working process according to the activity flow. After the activity of "inquires the questionnaire", according to whether inquires are succeed or not, which should be continued is decided, "inquires the questionnaire" activities or "show inquires the results" activities. When the inquires results are displayed, modifying questionnaire by implementing "changed the questionnaire" activities, then perform "save changed questionnaire to the database” activities, and save the amended results.

\section{Database Design}

The management of HIV's traditional Chinese medicine syndrome clinical information management system data based on Web is the core of the system, while there are also a variety of data, so, the first step of this system design research is designing for database system. According to the questionnaire content, they are divided into several database table objects, such as A type-general information, B type-medical project, C type-medical laboratory project, D type-interrogation (nearly 3 months), E type-inspection, F type-auscultation and olfaction, 
G type-palpation, H type-western medicine diagnosis, I typetraditional Chinese medicine syndrome differentiation.

Take relevant data table of patients as an example, see Table 1.

TABLE 1. PATIENTS’ BASIC INFORMATION FORM

\begin{tabular}{|c|l|l|l|l|}
\hline NO. & variable & type & If Null & explain \\
\hline 1 & NO & CHAR(8) & NOT NULL & $\begin{array}{l}\text { Id number (the } \\
\text { primary key number) }\end{array}$ \\
\hline 2 & A11 & CHAR(1) & NULL & Respondents group \\
\hline 3 & A12 & CHAR(1) & NULL & Living environment \\
\hline 4 & A13 & CHAR(1) & NULL & gender \\
\hline 5 & A14 & CHAR(1) & NULL & national \\
\hline 6 & A15 & DATE & NULL & Data of birth \\
\hline 7 & A16 & CHAR(1) & NULL & Marital status \\
\hline 8 & A17 & CHAR(1) & NULL & Cultural degree \\
\hline 9 & A18 & CHAR(1) & NULL & profession \\
\hline
\end{tabular}

The name of variable names are in consistent with that of the questionnaire's, the varieties are named by category, for example, in general situation, A type of variable names start with A. So that it is convenient to analyze and manage after the data is output to statistical analysis software as SPSS.

\section{The Realization and Test of the System}

\section{Three-layer Structure of the System}

Figure 6 is a three-layer system structure used in Internet database application, and also the system structure used in AIDS traditional Chinese medicine clinical information management system.

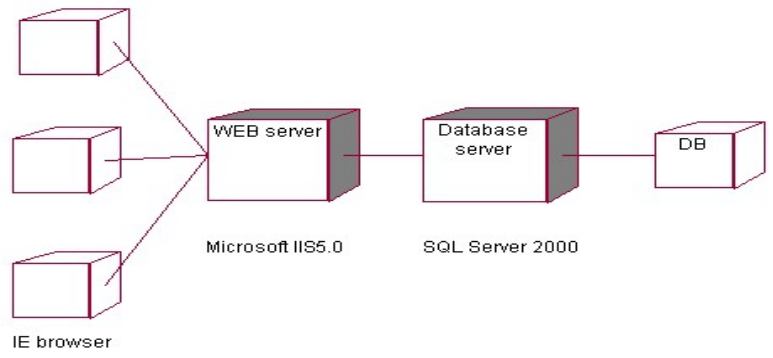

Figure 6. Three-layer system structure

Figure 6 shows the three processor of the Brower/Server structure: the browser or client computer, WEB server, database server. And can run different operating systems, the browser can run Windows XP, Vista operating system, etc. Both WEB server and database server can run Windows 2003 Advance Server. The browser is a HTTP client, produces requests to pages or other activities; and also provides script environment of client end. The browser instantiates views, turns them into HTML or other markup languages and displays on customers' browser windows. Web server handles HTTP protocol, receives requests, and generates response according to HTTP format, it also accepts scripts, we can use such languages like VBScript and JavaScript to write the code executed on the server. Web server can build, read, modify and delete database application view. The interface between Web server and database server transfers the SQL statement and relationship data, transmits Web page, the client code and data. Database server is responsible for the operation of DBMS, thus the SQL statement can be dealt with, and database management work can be executed.

UML is not only the model of demand analysis and system design; but also plays a key role in each stage of the software development process. According to the steps of RUP listed above, the main model of system has been established. In the following we will discuss the content of system realization and testing method.

The main realization interface of the system uses ASP technology to realize the function of each module. Software interface includes information input, inquiry, account management, system management, help, and other function menu.

\section{APPLICATION EFFECTS}

We have put the traditional Chinese medicine syndromes basic information data of 1654 AIDS patients into the system, and have made the corresponding statistical analysis in view of the investigation data. We have provided practical and efficient work platform among doctors, patients and scientific researchers ${ }^{[6,7]}$.

The systematization and standardization of patients management have been realized, the dynamic management of information of patients' each stage has been realized, which will bring a beneficial effect on the systematical treatment of AIDS.

The results show that the system is flexible and convenient, so it reaches to the design target. The establishment of the system will provide a feasible method for systematically studying AIDS traditional Chinese medicine syndromes information. There will be a positive meaning to prevent and cure AIDS, and the social benefit will be also significant. By studying the law of AIDS traditional Chinese medicine syndromes, the system makes specific therapy for AIDS patients in each period. Traditional Chinese Medicine is easy to be accepted by patients because of its low cost, small side effects, so it also has obvious economic benefits.

\section{CONCLUSION}

The results show that the system is flexible and convenient, so it reaches to the design target. The establishment of the system will provide a feasible method for systematically studying AIDS traditional Chinese medicine syndromes information. There will be a positive meaning to prevent and cure AIDS, and the social benefit will be also significant. By studying the law of AIDS traditional Chinese medicine syndromes, the system makes specific therapy for AIDS patients in each period. Traditional Chinese Medicine is easy to be accepted by patients because of its low cost, small side effects, so it also has obvious economic benefits

\section{REFERENCES}

[1] Shi ping Xie, "The ideas of traditional Chinese medicine for AIDS research,” Journal of Henan University of Traditional Chinese Medicine, vol. 2, Jan. 2005, pp. 1-4. 
[2] Xuan-xian Guo, Xie Shi ping, and Sun Lin, "Research situation about TCM etiology of AIDS," Chinese Medicine Research, vol. 20, March 2007, pp. 44-59.

[3] Jian zhong Guo and Xu Li ran, "The quantitative thinking and analysis about Traditional Chinese medicine clinical symptoms of AIDS," Journal of Henan University of Traditional Chinese Medicine, vol. 22, vol. May 2007, pp.5-10.

[4] Shiji Chen, "The design and implementation of intelligent elective course system based on UML modeling technology," Computer And Modernization, vol. 2, Feb. 2007, pp. 46-49.
[5] X. H. Liu, The base of UML and Visio modeling, Electronic Industry Press, 2004.

[6] Song He Shi, Xue Zhong Shi, and Ying Yan Li, Computer application in medical data analysis, Lan ZhouL: Lan Zhou university press, 2005.

[7] Lin Lin Li, Song He Shi, "The principal component analysis of HIV/AIDS patients based on four diagnostic information of traditional Chinese medicine," Journal of Zheng Zhou University (medical sciences), vol. 42, April 2007, 679-684. 